\title{
The Perinucleolar Compartment
}

\author{
Callie Pollock and Sui Huang \\ Northwestern University Feinberg School of Medicine, Chicago, Illinois 60611 \\ Correspondence: s-huang2@northwestern.edu
}

The perinucleolar compartment (PNC) is a subnuclear body characterized by its location to the periphery of the nucleolus. The PNC is a dynamic structure and is highly enriched in RNA-binding proteins and pol III RNA. The structural stability of the PNC is dependent on continuous pol III transcription and the presence of key proteins. The PNC is associated with malignancy both in vitro and in vivo and its presence positively correlates with metastatic capacity, making it a potential cancer marker. Recent studies also suggest an association between the PNC and a specific DNA locus, and ongoing PNC research continues to focus on determining the structure and function of the PNC to understand its role in cancer. This article summarizes the current understanding of PNC structure and function with an emphasis on the association of PNC and malignancy.

$T^{\text {he }}$ he cell nucleus is a complex and highly organized organelle that is responsible for many critical cellular functions, including housing and replicating DNA, transcribing DNA into RNA, RNA processing and splicing, macromolecular trafficking between the nucleus and cytoplasm, and regulating gene expression. Increasing evidence shows that these functions are not randomly distributed in the nucleus, but rather are highly compartmentalized into a higher order three-dimensional organization. Such organization is represented by nonmembrane-bound nuclear domains and nuclear bodies, which can be identified and visualized through various molecular probes and resolved by microscopy. The compartmentalization within the nucleus may reflect the highly efficient spatial and temporal organization of essential molecular complexes for specific functions (reviewed in Zimber et al. 2004).
Nucleoli, Cajal bodies (Morris 2008), promyelocytic leukemia (PML) bodies (Bernardi and Pandolfi 2007), nuclear speckles (Lamond and Spector 2003), and perinucleolar compartments (PNCs) are among the many nuclear compartments that have distinct nuclear localization patterns. As ongoing research examines the structure and function of these bodies, their functional relevance is being revealed. For example, nucleoli are involved in the biogenesis of ribosomes; nuclear speckles are involved in pre-mRNA processing and metabolism; Cajal bodies are indicated in the assembly and trafficking of snRNP, snoRNP, and telomere functions; and PML as well as PNC are associated with malignancy. This article summarizes the current understanding of the structure and function of one nuclear body, the PNC, and its role in the malignant phenotype.

Editors: David Spector and Tom Misteli

Additional Perspectives on The Nucleus available at www.cshperspectives.org

Copyright (C) 2010 Cold Spring Harbor Laboratory Press; all rights reserved; doi: 10.1101/cshperspect.a000679

Cite this article as Cold Spring Harb Perspect Biol 2010;2:a000679 


\section{PNC STRUCTURE}

The perinucleolar compartment (PNC) is a unique subnuclear structure that was originally described during the characterization of the hnRNP I/PTB (polypyrimidine tract binding) protein, in which the protein was shown to localize at a perinucleolar focus (Ghetti et al. 1992). The PNC is irregularly shaped, ranging from .25 to $4 \mu \mathrm{m}$ in length, and is associated with the periphery of the nucleolus (Matera et al. 1995; Huang et al. 1997). Electron microscopic analysis of HeLa cells reveals that the PNC is composed of multiple thick, electron dense strands, each measuring approximately $80-180 \mathrm{~nm}$ in diameter. The PNC is physically associated with the nucleolus while remaining structurally distinct (Fig. 1) (Huang et al. 1997). A three-dimensional computer reconstruction of electron microscopic images from serially sectioned HeLa cell nuclei shows that the PNC forms a reticulated mesh on the nucleolar surface (Huang et al. 1998).

Time lapse microscopy of cells expressing green fluorescent protein-tagged polypyrimidine tract-binding protein (GFP-PTB) shows that the PNC is a dynamic structure that moves discretely along the nucleolar periphery over time (Table 1) (Huang et al. 1997). PNC structure is maintained throughout interphase, disassembled during mitosis in conjunction with the disassociation of nucleoli, and is reassembled at late telophase as nucleoli begin to assemble. The PNC is commonly heritable from mother to daughter cells, and the PNCs of daughter cells are of similar shape, size, and number (Huang et al. 1997).

\section{MOLECULAR COMPONENTS OF THE PNC}

Although the composition of the PNC has not been fully characterized, a number of proteins and RNAs show enrichment in the PNC. All known PNC-associated proteins are RNAbinding proteins that are implicated in pol II RNA metabolism. The proteins include CUG binding protein (CUG-BP) (Timchenko et al. 1996), Polypyrimidine tract-binding protein (PTB) (Ghetti et al. 1992), KH-type splicing regulatory protein (KSRP) (Hall et al. 2004), Raver1 (Huttelmaier et al. 2001), Raver2 (Kleinhenz et al. 2005), Rod1, and nucleolin (Kopp and Huang 2005). Interestingly, the RNAs identified in the PNC so far are small, noncoding RNAs transcribed by pol III, which is surprising because pol II RNAs commonly interact with the PNC proteins. RNAs that show enrichment in the PNC include RNase mitochondrial processing (MRP) RNA, ribonuclease P (RNase P) RNA, hY $(1,2,5)$ RNA (Matera et al. 1995), Alu RNA, and signal recognition particle (SRP) (7SL) RNA (Wang et al. 2003).

\section{PNC-associated Proteins}

Proteins of diverse cellular functions are enriched in the PNC. What these proteins have in common is their primary implication in the processing of pol II RNAs. For example, the first protein identified in the $\mathrm{PNC}, \mathrm{PTB}$, is involved

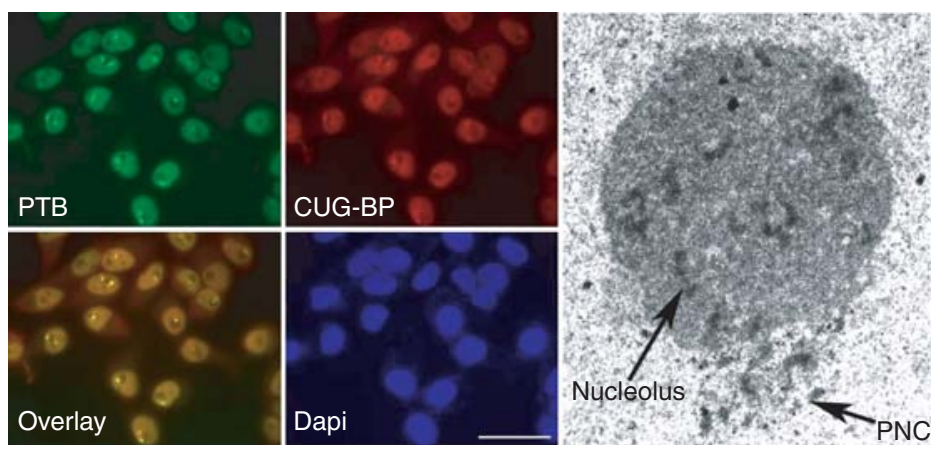

Figure 1. PNC localization. A specific PTB monoclonal antibody, SH54, immunolabels the perinucleolar compartment (PNC) in green, and CUG-BP is immunolabeled in red. In the far right panel, an electron microscopic image shows the PNC at the periphery of the nucleolus. Bar, $30 \mu \mathrm{m}$. 
The PNC

Table 1. Protein and RNA components of the PNC

\begin{tabular}{|c|c|}
\hline Component Name & Brief Description \\
\hline \multicolumn{2}{|l|}{ Protein components } \\
\hline CUG-BP & $\begin{array}{l}\text { Binds polyadenylated RNA and is involved in alternative splicing, deadenylation, and } \\
\text { mytonic dystrophy (Timchenko et al. 1996; Savkur et al. 2001; Ho et al. 2005) }\end{array}$ \\
\hline KSRP & $\begin{array}{l}\text { Involved in alternative splicing, splicing regulation, and RNA decay (Min et al. 1997; } \\
\text { Nechama et al. 2008) }\end{array}$ \\
\hline Nucleolin & $\begin{array}{l}\text { Enriched in the nucleolus and PNC, plays a role in rDNA transcription and RNA } \\
\text { processing (Ginisty et al. 1999; Tuteja and Tuteja 1998) }\end{array}$ \\
\hline PTB & $\begin{array}{l}\text { Protein involved in pre-mRNA splicing, alternative splicing, RNA polyadenylation, and } \\
\text { translation (Lou et al. 1996; Stoneley and Willis 2004; Wagner and Garcia-Blanco 2001) }\end{array}$ \\
\hline Raver $1 / 2$ & RNA binding, nuclear RNPs that interact with PTB (Kleinhenz et al. 2005) \\
\hline ROD1 & $\begin{array}{l}\text { Shares homology with PTB and is a homolog to yeast regulator of differentiation } \\
\text { (Yamamoto et al. 1999) }\end{array}$ \\
\hline \multicolumn{2}{|l|}{ RNA Components } \\
\hline Alu RNA & $\begin{array}{l}\text { Forms Alu complex that is implicated in regulation of transcription and translation } \\
\text { (Hasler and Strub 2006a; Hasler and Strub 2006b) }\end{array}$ \\
\hline hY RNA & Interacts with Ro protein to form Ro ribonucleoproteins (RNPs) (Wolin and Steitz 1984) \\
\hline MRP RNA & $\begin{array}{l}\text { RNA portion of the MRP RNP, which plays a role in mitochondrial DNA replication and } \\
\text { preribosomal RNA processing (van Eenennaam et al. 2000) }\end{array}$ \\
\hline RNase P RNA & $\begin{array}{l}\text { RNA component of RNase P complex, involved in maturation of } 5^{\prime} \text { end of tRNA (van } \\
\text { Eenennaam et al. 2000) }\end{array}$ \\
\hline SRP RNA & $\begin{array}{l}\text { Part of the signal recognition particle, implicated in secretory protein transport and } \\
\text { elongation arrest (Wolin and Walter 1989) }\end{array}$ \\
\hline
\end{tabular}

in pre-mRNA splicing (Wagner and GarciaBlanco 2001), alternative splicing, and regulation of RNA polyadenylation (Lou et al. 1996) and translation (Stoneley and Willis 2004). Another protein enriched in the PNC, CUG-BP, is known for its role in myotonic dystrophy neuromuscular disease, and it is also involved in alternative splicing and deadenylation (Timchenko et al. 1996; Savkur et al. 2001; Ho et al. 2005). KSRP has been implicated in alternative splicing and regulation of splicing (Min et al. 1997; Nechama et al. 2008). The enrichment of RNA processing proteins in the PNC suggests a possible role for the PNC in RNA metabolism. However, not all pre-mRNA processing factors, such as SR proteins, are enriched in the PNC. In addition, many attempts to localize pre- or mRNA have failed to observe any enrichment of pol II RNA in the PNC. Although the PNC is physically associated with the nucleolus, many nucleolar proteins are not enriched in the PNC. The one nucleolar protein found enriched in the PNC is nucleolin (Kopp and Huang 2005).

\section{PNC-associated RNAs}

A subset of pol III transcripts are enriched in the PNC. RNase P and MRP RNA (Matera et al. 1995) are two highly conserved components of sequence-specific endoribonucleases. As part of the endoribonuclease complexes, RNase $\mathrm{P}$ is involved in tRNA maturation, and RNase MRP is implicated in pre-ribosomal RNA processing and mitochondrial DNA replication (van Eenennaam et al. 2000). Furthermore, RNase P and MRP RNA are structurally related to each other and share protein binding partners in their functional endoribonuclease complexes. It is not known if this similarity is involved in the coenrichment of these RNAs to the PNC. Other PNC-enriched RNAs include hY RNA, an abundant RNA that associates with the Ro protein and may be involved in DNA replication (Wolin and Steitz 1984; Christov et al. 2006), and SRP RNA, an RNA component of the signal recognition particle, which functions in secretory protein transport and elongation arrest (Wolin and Walter 1989; Grudnik et al. 2009). 
Alu RNA also localizes to the PNC and is known to associate with the signal recognition particle (Hasler and Strub 2006b). Although only pol III transcripts have been detected in the PNC, not all pol III transcripts are enriched in the PNC. In situ hybridization of many pol III transcripts including U6, tRNA, and 7SK do not show localization to the PNC (Matera et al. 1995; Pollock and Huang 2009).

\section{Formation of the PNC}

The PNC is enriched with pol III transcribed RNAs and RNA binding proteins that are primarily implicated in the metabolism of pol II RNAs. The enrichment of the RNA and proteins in the PNC does not appear to be due to overexpression of these components because overexpression of the protein components ( $\mathrm{PTB}$ or CUG-BP) alone or with PNC-associated RNAs (MRP RNA and RNase P RNA) does not induce the formation of the PNC in primary or immortalized cells (Kopp and Huang 2005). Many of the molecular components of the PNC have been identified and the interactions amongst the known PNC-associated components are beginning to be investigated. It is known that some of the PNC-associated proteins interact with one another and the pol III RNAs that enrich in the PNC. For example, PTB interacts with Raver1 (Huttelmaier et al. 2001), nucleolin (Singh et al. 2004), and some of the hY RNAs (Fabini et al. 2001). It is not known, however, if these interactions occur within the PNC or elsewhere in the cell.

\section{PNC AND RNA TRANSCRIPTION}

RNA is crucial to PNC stability. This importance of RNA was first noticed when RNase, but not DNase, treatment eliminated PNC in permeabilized cells (Huang et al. 1998). A 5 minute pulse-labeling of $\mathrm{Br}-\mathrm{U}$ showed that a majority of PNC-associated RNA is newly synthesized (Huang et al. 1998). To determine the origin of PNC-enriched RNA, pol I, II, or III transcriptional activities were selectively inhibited. Initial experiments using actinomycin $\mathrm{D}$ at a low concentration that preferentially inhibits pol I transcription showed that PNC structure is disrupted by the treatment. However, a later experiment using cycloheximide treatment, a protein translation inhibitor that also inhibits pol I transcription $(5 \mathrm{~h}$ at $100 \mu \mathrm{g} / \mathrm{mL}$ ) (Higashi et al. 1968), did not induce PNC disassembly or affect labeled nucleotide enrichment in the PNC, demonstrating that pol I transcription is not important to PNC structure. The sensitivity of the PNC to actinomycin D is most likely because of the multiple modes of action of the drug, including its DNA interaction, which directly affects PNC structure (see below). Additionally, pol I transcripts (i.e., $28 \mathrm{~S}$ and $18 \mathrm{~S}$ RNAs) or prerRNA processing factors, such as fibrillarin, are not detected in the PNC (Matera et al. 1995; Huang et al. 1997; Kopp and Huang 2005). These results suggest that PNC-enriched RNAs are not likely to be of pol I origin. Nor is it likely that the primary source of PNCenriched RNA is from RNA pol II because treatment of $\alpha$-amanitin, a drug that selectively and specifically inhibits pol II polymerase, does not induce PNC dissociation (Huang et al. 1998). In addition, in situ hybridization to several pre-mRNAs did not show coenrichment of these RNA to the PNC (Hall et al. 2004). However, it can not be excluded, at this point, that a small subset of pol I and II transcripts may associate with the PNC.

The structural integrity of the PNC is dependent on pol III transcription. Injection of tagetin, a specific inhibitor of polymerase III, causes disassembly of the PNC within 2 hours (Wang et al. 2003). Furthermore, the continuous production of pol III transcripts, not just pol III activity, is important for PNC structure. Overexpression of RNase MRP RNA from a pol II promoter was able to partially overcome the PNC instability caused by pol III inhibition (Wang et al. 2003). PNC-enriched pol III RNAs are most likely newly transcribed. A 5 minute pulse-label with $\mathrm{BrU}$ shows that newly synthesized RNAs are coenriched in the PNC with the RNA binding proteins (Huang et al. 1998). In addition, during initial pol III inhibition, PNC-associated MRP RNA rapidly dissociates, whereas mature pol III RNAs in 
their functional complexes remain unaltered. For example, nucleolar-associated functional MRP RNA remains in the nucleolus at similar time points (Wang et al. 2003). These results reveal that the pol III transcripts enriched in the PNC are not likely to be in their mature, functional complexes, and these RNAs are necessary for the maintenance of PNC structure.

Although the PNC is enriched with newly transcribed pol III RNAs, it is not the site of transcription for these RNAs. In situ hybridization of genes encoding four PNC-associated RNAs shows no spatial proximity to the PNC (Matera et al. 1995; Kopp and Huang 2005). In addition, it is also unlikely that the PNC acts as an assembly point for the newly transcribed RNAs into their respective RNP complexes, as the protein subunits of these complexes are not detected to be similarly enriched in the PNC (Hall et al. 2004; Kopp and Huang 2005).

Although the specific function of the PNC remains unidentified, the enrichment of newly synthesized pol III RNAs and RNA binding proteins suggests a role in RNA metabolism. Several potential functions of the PNC can be envisioned. First, the PNC may represent a transitional depot between the newly synthesized pol III RNA and the assembly of their final functional complex. Second, the PNC may be a site of RNA processing. Third, the PNC could act as a site of assembly for RNA trafficking complexes for movement to their functional destinations, including those in the nucleus or in the cytoplasm. Fourth, the PNC may be a site for degradation of excess pol III RNAs that are produced because of the pol III deregulation often observed in cancer cells. Because live cell studies have shown that PTB and CUG-BP shuttle in and out of PNCs rapidly (Huang et al. 1997), the association of these RNAs with the PNC is most likely dynamic rather than simple aggregation or long-term storage.

\section{PNC ASSOCIATION WITH DNA}

Initial characterization of the PNC showed that daughter cells inherit PNCs often arranged as mirror images from their parent cells (Huang et al. 1997). This heritable nature of the PNC suggested a connection between the structure and DNA, and prompted further investigation into the association. More recent chemical and cell biology experiments show that PNC stability is reliant on DNA integrity, and the PNC is associated with a DNA locus.

\section{Chemical Biology Evidence}

To better understand the structure and function of the PNC, a screen was designed to identify compounds that disassemble the PNC, and through this study a large array of genome-toxic drugs were found to dissociate PNCs (Norton et al. 2009). These drugs work through three distinct modes of action: pol III transcription inhibition, DNA damage, or both. A pol III promoter assay in cells treated with various compounds that influence pol III transcription shows that PNC prevalence reduction corresponds to the level of transcription inhibition induced by the drugs (Norton et al. 2009), which is consistent with a previous finding that pol III transcription is essential for the PNC integrity (Wang et al. 2003).

To determine the mechanism by which DNA damage disassembles PNCs, the role of DNA damage repair on PNC structure was evaluated. Blocking the repair response pathway through either specific inhibitors or siRNA to the DNA damage signaling kinases ATM, ATR, and Chk2 does not block the reduction of the PNC after DNA damage, but does block the recovery of the structure after DNA damage (Norton et al. 2009). This suggests that the loss of PNC stability is a direct result of DNA damage, not an effect of the DNA damage response pathway. Additionally, not all types of DNA damage disrupt the PNC. Although DNA intercalators, topisomerase inhibitors, UV treatment, and cross linkers reduce PNC, double strand or single strand breakers, reactive oxygen species, or alkalators have no impact on PNC structure. The effective PNC disassembly compounds all are able to disrupt the base-pairing capacity of DNA, suggesting that the integrity of DNA is critical to the PNC structure. Furthermore, treatment with a histone deacetylase inhibitor (TSA), which favors an open 
chromatin conformation, also changes the PNC from a dense, round structure into an extended fibril structure. This finding indicates that the PNC-associated locus is responsive to the epigenetic regulations (Norton et al. 2009).

\section{Cell Biology Evidence}

Cell biological studies, using several approaches, further support the association of the PNC with a DNA locus. When a conditional mutant of the cell cycle kinase Cdk1 is grown at a nonpermissive condition, DNA undergoes continuous replication without cell division. The number of PNC per cell increases in parallel with the number of replication cycles in these endoreplicating cells, indicating that the number of PNC per cell is directly linked to the copy number of the DNA (Norton et al. 2009). Examination of synchronized HeLa cells shows that during $S$ phase, PNCs generally split into a doublet predominantly at 4-6 hours into replication, but become one again during G2 phase. This is consistent with the behavior of a DNA locus during replication and implies that the PNC-associated locus replicates at mid S phase (Norton et al. 2009). Together with the finding that the PNC extends into a fibril structure on treatment with TSA, these studies firmly indicate the association of the PNC with a specific DNA locus.

Although recent studies suggest a specific PNC-DNA association, the exact locus with which the PNC interacts has yet to be determined. It is possible that the DNA locus may represent the transcription site for a previously unidentified PNC-associated RNA, or alternatively the PNC could nucleate on a specific locus and regulate expression. Research is ongoing to determine the PNC associating DNA locus.

\section{PNC AND TRANSFORMATION}

Initial characterization of the PNC showed that the PNC is predominantly present in cancer cells (Huang et al. 1997). A more complete investigation of over 50 cancerous and normal cell lines confirmed this trend (Norton et al. 2008). PNC prevalence (\% cells containing one or more PNC) is consistently low $(<5 \%)$ in normal cell lines and immortalized cell lines $(0 \%-6 \%)$ derived from multiple human and mouse tissue types including stromal, endothelial, hematopoietic, or embryonic stem cells. PNC prevalence heterogeneously increases (15\% to near $100 \%$ ) in cancer cell lines. PNCs form in carcinomas, blastomas, and sarcomas, but PNC prevalence is not significantly elevated in malignant cell lines of hematopoietic origin. This suggests that the PNC selectively forms in malignant cells derived from solid tumor tissues (Norton et al. 2008).

\section{In Vivo Studies}

The strong correlation of PNC prevalence with malignancy in vitro prompted in vivo investigations. PNC prevalence was evaluated in paraffin-embedded human breast cancer tissues in varying clinical stages. Histological samples of normal breast tissue, primary tumors, affected lymph nodes, and distant metastasis were scored for PNC prevalence. The results showed that PNC prevalence is $0 \%$ in normal breast tissue, but it increases in the primary tumor $(47.9 \%)$ and affected lymph nodes $(76.3 \%)$, and reaches near $100 \%$ in distant metastases (Kamath et al. 2005). Additionally, high PNC prevalence in primary tumors of stage I patients positively correlates with disease relapse in a case-matched study, and it is predictive of survival in a retrospective 17-year followup study (Kamath et al. 2005). The correlation between PNC prevalence and metastasis suggests that the PNC-containing cells have a metastatic advantage over non-PNC-containing cells. The formation of the PNC may reflect key changes during transformation that are associated with metastatic capability.

\section{PNC and Metastasis}

To address the association of PNC prevalence and metastasis, cell lines of varying metastatic potential have been examined. Studies using a well characterized prostate cancer metastasis model (Pettaway et al. 1996) show that PC-3M cells, which are enriched for metastatic capacity, 
have a much higher PNC prevalence (85\%) than the parental PC-3 heterogenous population (4\%). Furthermore, additional rounds of selection for metastatic capacity led to a further increase in PNC prevalence. For example, the PC-3M LN4 cell line, which undergoes an additional four rounds of selection for metastatic capacity, has a PNC prevalence of 98\% and abnormally large PNCs. In contrast, PC-3M Pro 4 cells, which are PC-3M cells that have gone through four rounds of selection for a lack of metastatic capacity, show a marked reduction in PNC size and a small reduction in PNC prevalence (71\%) compared with PC-3M cells (85\%) (Norton et al. 2008). Moreover, when the PNC prevalence is adjusted to the percentage of cells with PNCs greater than 2.2 $\mu \mathrm{m}$, it correlates very closely with the metastatic behavior of these cells. These observations in cells of the same origin, but of varying metastatic capacities, further confirm that PNC prevalence reflects the metastatic capability of cancer cells.

The association of PNC with metastatic capacity is not limited to prostate cancer. To evaluate the association of PNC prevalence with metastatic behavior in another system, two cell lines have been created from a single patient: one from a primary melanoma tumor and one from a distant metastasis. PNC prevalence increases dramatically in cell lines derived from distant metastases over those derived from the primary tumor, and the same results are observed in a similar experiment using colorectal cell lines (Norton et al. 2008). Additionally, PNC prevalence has been examined in cell lines overexpressing the breast cancer related metastatic suppressor protein (BRMS). BRMS is a chromatin remodeling protein that suppresses the ability of breast cancer cells to metastasize (Samant et al. 2000). Stable overexpression of this protein in two breast cancer cell lines significantly reduces metastasis compared with the parental cells when injected into a nude mouse (Samant et al. 2000). The PNC prevalence in the BRMS overexpressing cell lines is significantly lower than in the parental cells, further confirming the association of the PNC with metastatic cells (Norton et al. 2009).
The unique association of the PNC with metastatic cancer cells from solid tumors suggests that it forms in connection with cellular conditions specific to these cells. Multiple traits common to cancer cells, such as increased proliferation and glycolysis, have been examined for an association with PNC prevalence. Results show that PNC prevalence is not affected by cell proliferation. HeLa cells grown in serum-free media show a significantly reduced growth rate, but PNC prevalence does not change. High glucose or low glucose growth conditions do not alter PNC prevalence, which shows the glycolysis state does not influence PNC formation. Furthermore, inducing changes in differentiation status of cancer cells does not affect PNC prevalence, and PNCs are not present in embryonic stem cells (Norton et al. 2008). These results support the idea that PNC formation selectively associates with metastatic behavior.

Potential of Clinical Applications for the PNC

Recent studies propose the use of the PNC as a cancer marker for solid tumors. The PNC is an easily scored, multicomponent structure that reflects the complex cellular conditions that develop in the later stages of malignant transformation. The PNC is unique in that it is selectively associated with metastasis without known links to proliferation, glycolysis, or differentiation state. Selective and specific tumor markers could help make appropriate treatment decisions for many cancer patients. Currently, histological PNC prevalence scoring in tissue samples is being refined to improve its reproducibility and reliability as a marker.

The PNC has also been presented as an anticancer drug discovery marker. The PNC is associated with the metastatic capacity of cells, and therefore PNC elimination could indicate a shift of cellular behavior from a higher malignant toward a more benign phenotype. In this way, the efficacy of drugs as anticancer agents could be screened for their ability to reduce PNC prevalence. Clinically used cancer drugs and experimental cancer drugs have been screened and a large number of these drugs 
effectively reduce PNC prevalence (Norton et al. 2009). This validates the potential use of PNC prevalence reduction as a phenotypic screening strategy to identify novel compounds that selectively inhibit malignant cells. Compounds that eliminate the PNC not only have potential to be developed into novel drugs, but can also be used as chemical biology tools to help understand the structure and function of the PNC and its functional significance in cancer cells.

\section{PNC FUNCTION IN MALIGNANCY}

The function of the PNC in malignancy remains to be investigated. However, the close association with cells of metastatic capability suggests that the PNC forms in response to the transformation process, possibly allowing cells to best adapt to a new physiological condition. In other words, the PNC may form as the consequence of transformation to better maintain or further promote the transformed phenotype. Thus, PNCs could play an active role in regulation of gene expression in these cells. As PNCs have been shown to be enriched in newly synthesized pol III RNA, the function of the PNC may be tied to the regulation of pol III RNA expression in malignant cells. The link of Pol III deregulation to cancer was first observed in mouse melanoma cells, where pol III is hyperactive (Schwartz et al. 1974), and more recent investigations support these findings in human cell lines and tissues (Liebhaber et al. 1978; Winter et al. 2000). A recent study showed that the overexpression of the pol III transcripts, tRNA or 5S rRNA alone, is sufficient to cause increased proliferation and oncogenic transformation (Marshall et al. 2008). These findings suggest that increased pol III transcription not only correlates with malignancy, but may play a direct role in the development or progression of cancer. Although PNC-enriched pol III transcripts have not been shown to have a direct role in the development of cancer, the concentration of pol III transcripts in the PNC could be indicative of the deregulation of these RNA in cancer cells. The nucleation of pol III transcripts at the PNC may be involved in regulating the function of these RNAs in cancer progression or maintenance. Studies are ongoing to investigate the complexes that pol III RNAs form in association with the PNC and their functional relevance to malignancy.

There are a number of additional possibilities regarding the potential functions of PNC formation in malignancy. First, yet to be identified factors in the PNC might be altered in solid tumor cells. The increased expression or altered function of these components may lead to nucleation of the RNAs and RNA-binding proteins in the PNC, which could directly influence the availability of PNC-associated RNA or proteins in cancer cells. Second, the PNC may play a role in nucleolar function, which is known to be deregulated in malignant cells (reviewed in Ruggero and Pandolfi 2003). The PNC is in close spatial proximity to the nucleolus, and the PNC is enriched in components (i.e., Nucleolin, PTB, RNase MRP RNA, and RNase P RNA) that are involved in rDNA transcription and ribosome synthesis. Studies are underway to determine the role of the PNC in malignancy.

\section{SUMMARY}

Although the function of the PNC is not yet fully understood, significant progress has been made in the characterization of the PNC in recent years. The PNC is a unique and dynamic nuclear body that selectively associates with metastatic capacity in a broad array of solid tumor tissues. The PNC is enriched in both RNA-binding proteins and a subset of newly synthesized pol III transcripts. Furthermore, the PNC is nucleated on a DNA locus or loci, and the integrity of DNA is critical for the PNC structure. The current working model is that the PNC is associated with and may represent changes in novel molecular complexes that are involved in the metabolism of newly synthesized pol III RNA during malignancy. Nucleation of the PNC on a DNA locus may be directly involved in the regulation of gene expression at that locus. Studies are underway to identify and characterize novel pol III-RNA complexes and the DNA locus they nucleate 
on to form the PNC. These investigations will help elucidate the role of the PNC and increase our knowledge of basic cancer biology.

\section{ACKNOWLEDGMENTS}

S.H. is funded by R01 GM078555-01A1 and C.P. is funded by the T32 CA080621-06A2 training grant.

\section{REFERENCES}

Bernardi R, Pandolfi PP. 2007. Structure, dynamics and functions of promyelocytic leukaemia nuclear bodies. Nat Rev Mol Cell Biol 8: 1006-1016.

Christov CP, Gardiner TJ, Szuts D, Krude T. 2006. Functional requirement of noncoding Y RNAs for human chromosomal DNA replication. Mol Cell Biol 26: 6993-7004.

Fabini G, Raijmakers R, Hayer S, Fouraux MA, Pruijn GJ, Steiner G. 2001. The heterogeneous nuclear ribonucleoproteins $\mathrm{I}$ and $\mathrm{K}$ interact with a subset of the ro ribonucleoprotein-associated Y RNAs in vitro and in vivo. J Biol Chem 276: 20711-20718.

Ghetti A, Pinol-Roma S, Michael WM, Morandi C, Dreyfuss G. 1992. hnRNP I, the polypyrimidine tract-binding protein: Distinct nuclear localization and association with hnRNAs. Nucleic Acids Res 20: 3671-3678.

Grudnik P, Bange G, Sinning I. 2009. Protein targeting by the signal recognition particle. Biol Chem 390: 775-782.

Hall MP, Huang S, Black DL. 2004. Differentiation-induced colocalization of the $\mathrm{KH}$-type splicing regulatory protein with polypyrimidine tract binding protein and the c-src pre-mRNA. Mol Biol Cell 15: 774-786.

Hasler J, Strub K. 2006a. Alu elements as regulators of gene expression. Nucleic Acids Res 34: 5491-5497.

Hasler J, Strub K. 2006b. Alu RNP and Alu RNA regulate translation initiation in vitro. Nucleic Acids Res 34: 2374-2385.

Higashi K, Matsuhisa T, Kitao A, Sakamoto Y. 1968. Selective suppression of nucleolar RNA metabolism in the absence of protein synthesis. Biochim Biophys Acta 166: 388-393.

Ho TH, Bundman D, Armstrong DL, Cooper TA. 2005. Transgenic mice expressing CUG-BP1 reproduce splicing mis-regulation observed in myotonic dystrophy. Hum Mol Genet 14: 1539-1547.

Huang S, Deerinck TJ, Ellisman MH, Spector DL. 1997. The dynamic organization of the perinucleolar compartment in the cell nucleus. J Cell Biol 137: 965-974.

Huang S, Deerinck TJ, Ellisman MH, Spector DL. 1998. The perinucleolar compartment and transcription. J Cell Biol 143: 35-47.

Huttelmaier S, Illenberger S, Grosheva I, Rudiger M, Singer RH, Jockusch BM. 2001. Raver1, a dual compartment protein, is a ligand for $\mathrm{PTB} / \mathrm{hnRNPI}$ and microfilament attachment proteins. J Cell Biol 155: 775-786.

Kamath RV, Thor AD, Wang C, Edgerton SM, Slusarczyk A Leary DJ, Wang J, Wiley EL, Jovanovic B, Wu Q, et al. 2005. Perinucleolar compartment prevalence has an independent prognostic value for breast cancer. Cancer Res 65: 246-253.

Kleinhenz B, Fabienke M, Swiniarski S, Wittenmayer N, Kirsch J, Jockusch BM, Arnold HH, Illenberger S. 2005. Raver2, a new member of the hnRNP family. FEBS Lett 579: $4254-4258$.

Kopp K, Huang S. 2005. Perinucleolar compartment and transformation. J Cell Biochem 95: 217-225.

Lamond AI, Spector DL. 2003. Nuclear speckles: A model for nuclear organelles. Nat Rev Mol Cell Biol 4: 605-612.

Liebhaber SA, Wolf S, Schlessinger D. 1978. Differences in rRNA metabolism of primary and SV40-transformed human fibroblasts. Cell 13: 121-127.

Lou H, Gagel RF, Berget SM. 1996. An intron enhancer recognized by splicing factors activates polyadenylation. Genes Dev 10: 208-219.

Marshall L, Kenneth NS, White RJ. 2008. Elevated tRNA(iMet) synthesis can drive cell proliferation and oncogenic transformation. Cell 133: 78-89.

Matera AG, Frey MR, Margelot K, Wolin SL. 1995. A perinucleolar compartment contains several RNA polymerase III transcripts as well as the polypyrimidine tract-binding protein, hnRNP I. J Cell Biol 129: 1181-1193.

Min H, Turck CW, Nikolic JM, Black DL. 1997. A new regulatory protein, KSRP, mediates exon inclusion through an intronic splicing enhancer. Genes Dev 11: $1023-1036$.

Morris GE. 2008. The Cajal body. Biochim Biophys Acta 1783: $2108-2115$.

Nechama M, Ben-Dov IZ, Briata P, Gherzi R, Naveh-Many T. 2008. The mRNA decay promoting factor K-homology splicing regulator protein post-transcriptionally determines parathyroid hormone mRNA levels. FASEB J 22: 3458-3468.

Norton JT, Pollock CB, Wang C, Schink JC, Kim JJ, Huang S. 2008. Perinucleolar compartment prevalence is a phenotypic pancancer marker of malignancy. 113: 861-869.

Norton JT, Wang C, Gjidoda A, Henry RW, Huang S. 2009. The perinucleolar compartment is directly associated with DNA. J Biol Chem 284: 4090-4101.

Pettaway CA, Pathak S, Greene G, Ramirez E, Wilson MR, Killion JJ, Fidler IJ. 1996. Selection of highly metastatic variants of different human prostatic carcinomas using orthotopic implantation in nude mice. Clin Cancer Res 2: $1627-1636$.

Pollock C, Huang S. 2009. The perinucleolar compartment. J Cell Biochem 107: 189-193.

Ruggero D, Pandolfi PP. 2003. Does the ribosome translate cancer? Nat Rev Cancer 3: 179-192.

Samant RS, Seraj MJ, Saunders MM, Sakamaki TS, Shevde LA, Harms JF, Leonard TO, Goldberg SF, Budgeon L, Meehan WJ, et al. 2000. Analysis of mechanisms underlying BRMS1 suppression of metastasis. Clin Exp Metastasis 18: 683-693.

Savkur RS, Philips AV, Cooper TA. 2001. Aberrant regulation of insulin receptor alternative splicing is associated with insulin resistance in myotonic dystrophy. Nat Genet 29: $40-47$.

Schwartz LB, Sklar VE, Jaehning JA, Weinmann R, Roeder RG. 1974. Isolation and partial characterization of 


\section{Pollock and S. Huang}

the multiple forms of deoxyribonucleic acid-dependent ribonucleic acid polymerase in the mouse myeloma, MOPC 315. J Biol Chem 249: 5889-5897.

Singh K, Laughlin J, Kosinski PA, Covey LR. 2004. Nucleolin is a second component of the CD154 mRNA stability complex that regulates mRNA turnover in activated T cells. J Immunol 173: 976-985.

Stoneley M, Willis AE. 2004. Cellular internal ribosome entry segments: Structures, trans-acting factors and regulation of gene expression. Oncogene 23: 3200-3207.

Timchenko LT, Miller JW, Timchenko NA, DeVore DR, Datar KV, Lin L, Roberts R, Caskey CT, Swanson MS. 1996 Identification of a (CUG)n triplet repeat RNA-binding protein and its expression in myotonic dystrophy. Nucleic Acids Res 24: 4407-4414.

van Eenennaam H, Jarrous N, van Venrooij WJ, Pruijn GJ. 2000. Architecture and function of the human endonucleases RNase P and RNase MRP. IUBMB Life 49: 265-272.

Wagner EJ, Garcia-Blanco MA. 2001. Polypyrimidine tract binding protein antagonizes exon definition. $\mathrm{Mol} \mathrm{Cell}$ Biol 21: 3281-3288.
Wang C, Politz JC, Pederson T, Huang S. 2003. RNA polymerase III transcripts and the PTB protein are essential for the integrity of the perinucleolar compartment. Mol Biol Cell 14: 2425-2435.

Winter AG, Sourvinos G, Allison SJ, Tosh K, Scott PH, Spandidos DA, White RJ. 2000. RNA polymerase III transcription factor TFIIIC2 is overexpressed in ovarian tumors. Proc Natl Acad Sci 97: 12619-12624.

Wolin SL, Steitz JA. 1984. The Ro small cytoplasmic ribonucleoproteins: Identification of the antigenic protein and its binding site on the Ro RNAs. Proc Natl Acad Sci 81: 1996-2000.

Wolin SL, Walter P. 1989. Signal recognition particle mediates a transient elongation arrest of preprolactin in reticulocyte lysate. J Cell Biol 109: 2617-2622.

Yamamoto H, Tsukahara K, Kanaoka Y, Jinno S, Okayama H. 1999. Isolation of a mammalian homologue of a fission yeast differentiation regulator. Mol Cell Biol 19: 3829-3841.

Zimber A, Nguyen QD, Gespach C. 2004. Nuclear bodies and compartments: Functional roles and cellular signalling in health and disease. Cell Signal 16: 1085-1104. 


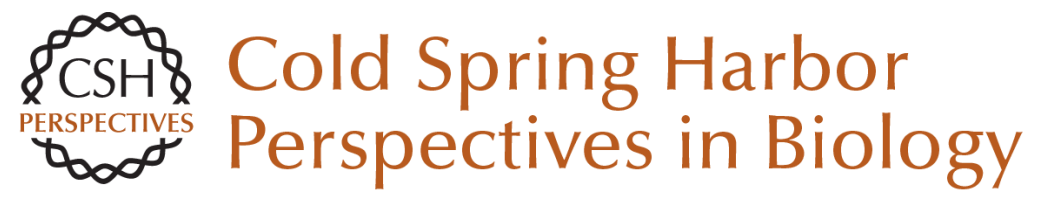

\section{The Perinucleolar Compartment}

Callie Pollock and Sui Huang

Cold Spring Harb Perspect Biol 2010; doi: 10.1101/cshperspect.a000679 originally published online January 20, 2010

\section{Subject Collection The Nucleus}

Nuclear Compartments: An Incomplete Primer to Nuclear Compartments, Bodies, and Genome Organization Relative to Nuclear Architecture Andrew S. Belmont

Uncovering the Principles of Genome Folding by 3D Chromatin Modeling

Asli Yildirim, Lorenzo Boninsegna, Yuxiang Zhan, et al.

3D or Not 3D: Shaping the Genome during Development Juliane Glaser and Stefan Mundlos

The Impact of Space and Time on the Functional Output of the Genome Marcelo Nollmann, Isma Bennabi, Markus Götz, et al.

Chromatin Mechanisms Driving Cancer

Berkley Gryder, Peter C. Scacheri, Thomas Ried, et al.

Liquid-Liquid Phase Separation in Chromatin Karsten Rippe

Mechanical Forces in Nuclear Organization Yekaterina A. Miroshnikova and Sara A. Wickström

Imaging Organization of RNA Processing within the Nucleus

Jeetayu Biswas, Weihan Li, Robert H. Singer, et al.
Mechanisms of Chromosome Folding and Nuclear Organization: Their Interplay and Open Questions Leonid Mirny and Job Dekker

Epigenetic Reprogramming in Early Animal Development

Zhenhai Du, Ke Zhang and Wei Xie

Essential Roles for RNA in Shaping Nuclear Organization

Sofia A. Quinodoz and Mitchell Guttman

The Molecular and Nuclear Dynamics of

$\mathrm{X}$-Chromosome Inactivation

François Dossin and Edith Heard

Structure, Maintenance, and Regulation of

Nuclear Pore Complexes: The Gatekeepers of the

Eukaryotic Genome Marcela Raices and Maximiliano A. D'Angelo

The Nuclear Lamina Xianrong Wong, Ashley J. Melendez-Perez and Karen L. Reddy

The Nuclear Pore Complex as a Transcription Regulator Michael Chas Sumner and Jason Brickner

Physical Nature of Chromatin in the Nucleus Kazuhiro Maeshima, Shiori lida and Sachiko Tamura

For additional articles in this collection, see http://cshperspectives.cshlp.org/cgi/collection/

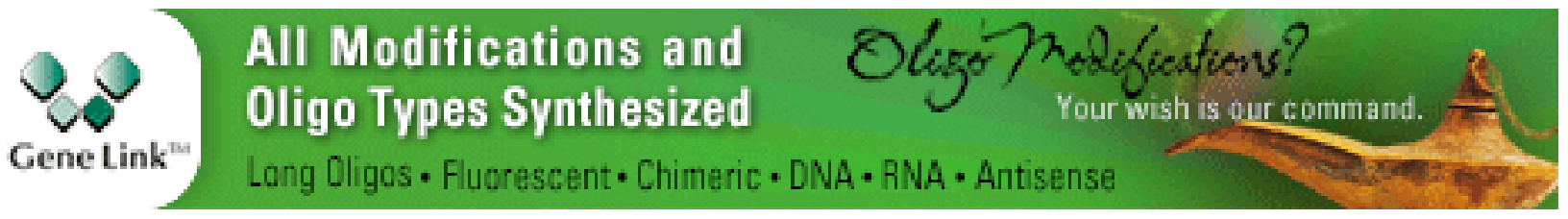


For additional articles in this collection, see http://cshperspectives.cshlp.org/cgi/collection/

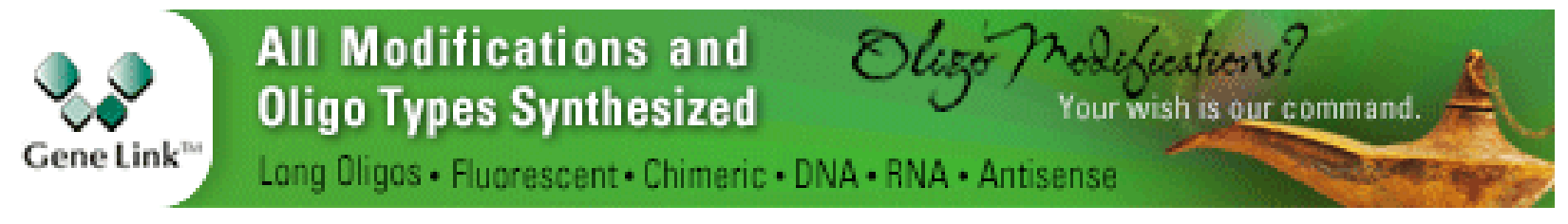

Copyright @ 2010 Cold Spring Harbor Laboratory Press; all rights reserved 